\title{
Reaction on a rink: Kondo-enhanced heterogeneous single-atom catalysis
}

\author{
Xiangyang Li, ${ }^{1}$ Hong Gong, ${ }^{1}$ Qingfeng Zhuang, ${ }^{1}$ Bing Wang, ${ }^{1}$ Xiao Zheng, $,{ }^{1,2}, *$ and Jinlong Yang ${ }^{1}, \dagger$ \\ ${ }^{1}$ Hefei National Laboratory for Physical Sciences at the Microscale 8 \\ Synergetic Innovation Center of Quantum Information and Quantum Physics, \\ University of Science and Technology of China, Hefei, Anhui 230026, China \\ ${ }^{2}$ CAS Center for Excellence in Nanoscience \& Key Laboratory of Surface and \\ Interface Chemistry and Energy Catalysis of Anhui Higher Education Institutes, \\ University of Science and Technology of China, Hefei, Anhui 230026, China
}

(Dated: February 26, 2021)

\begin{abstract}
Boosting the efficiency of heterogeneous single-atom catalysts (SACs) by adjusting the microenvironment of the active atom has recently attracted enormous attention. However, it remains largely unknown whether the atomic spin could be utilized to enhance the catalytic activity. We explore such a possibility by investigating the thermochemical effect of Kondo screening of a local atomic spin by free electrons in the metal support. Inspired by the exothermicity of Kondo spin-screening, a novel approach to heterogeneous catalysis - reaction on a rink (ROAR) - is proposed. Interestingly, in contrast to the conventional notion of thermal catalytic reaction, lowering the temperature of metal support is predicted to result in a reduced reaction barrier. As a proof of concept, CO oxidation catalyzed by the $\mathrm{Co@CoPc/Au(111)} \mathrm{composite} \mathrm{is} \mathrm{scrutinized} \mathrm{and} \mathrm{the} \mathrm{efficacy} \mathrm{of} \mathrm{ROAR}$ is demonstrated. This work accentuates the potential usefulness of the spin degrees of freedom to heterogeneous single-atom catalysis.
\end{abstract}

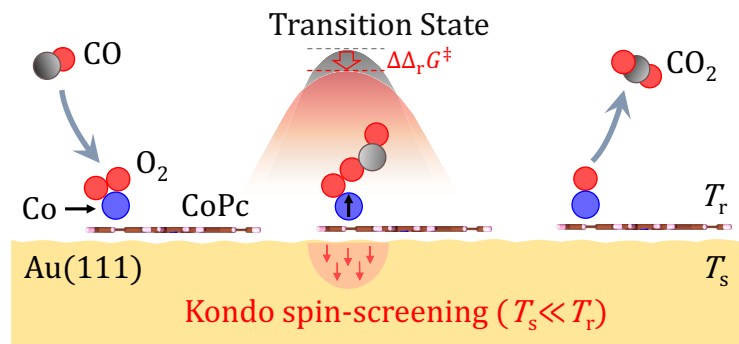

Keywords: single-atom catalysis, low temperature reaction, Kondo spin-screening, microenvironment, $\mathrm{CO}$ oxidation

\section{INTRODUCTION}

For thermal catalytic reactions, one of the most important goals is to reduce the energy barrier of the ratedetermining step, so that the reaction gets substantially accelerated and may occur under mild conditions. Recently, interests in heterogeneous single-atom catalysis have grown rapidly. ${ }^{1-3}$ This is not only because the atomically dispersed metal centers maximize the atom utilization efficiency ${ }^{4-6}$ but also due to the remarkable catalytic activity and selectivity which have been demonstrated in experiments. ${ }^{7-9}$

It has been discovered that the spin states of metal atoms may have critical influence on the performance of single-atom catalysts (SACs).$^{6,10-12}$ For instance, Liu et al. have reported that the intermediate-spin $\mathrm{FeN}_{5}$ species exhibits the highest activity and reusability among all the atomically dispersed $\mathrm{Fe}-\mathrm{N}-\mathrm{C}$ catalysts for the selective oxidation of $\mathrm{C}-\mathrm{H}$ bond. ${ }^{13}$ Moreover, Gong et al. have shown that, for the photocatalytic reduction of $\mathrm{CO}_{2}$ to $\mathrm{HCOOH}$, the covalent organic framework COF-367-Co ${ }^{\text {III }}$ with the embedded cobalt ions in the
$S=1 / 2$ state exhibits much higher activity and selectivity than the COF-367-Co ${ }^{\mathrm{II}}$ with $S=0$ cobalt ions. ${ }^{8} \mathrm{It}$ is thus appealing to design practical schemes to enhance the catalytic performance by exploiting the spin degrees of freedom of SACs.

Various strategies have been proposed to optimize the atomic microenvironment of $\mathrm{SACs},{ }^{14}$ e.g. by utilizing the synergistic effects of neighboring atoms, ${ }^{15,16}$ designing coordination ligands, ${ }^{17}$ tuning metal-support interaction, ${ }^{5,18}$ forming hydrogen bonding with surrounding atoms, ${ }^{19}$ etc. Despite the enormous efforts made, attempts to tune the spin-spin interaction between an SAC and its microenvironment have remained rather scarce. Some interesting questions can be raised, among which a fundamental one is: can the surrounding environment influence the local spin state of an SAC, and if so, can such influence be utilized to enhance the catalytic activity?

It is well-known that when a magnetic transition metal (TM) atom is adsorbed onto a metal support, the local spin moment on the TM adatom due to the spin-unpaired $d$-electrons can be screened by the itinerant electrons in 
the metal, provided that the temperature of metal support is low enough to suppress the thermal fluctuations of the free electrons. Such a spin-screening phenomenon is referred to as the Kondo effect, ${ }^{20}$ which results in the formation of strongly correlated electronic states at the TM adatom/metal interface, as has been affirmed by the conspicuous zero-bias resonant peaks in the scanning tunneling spectroscopy. ${ }^{21}$ Extensive works have been carried out to fine-tune the strength of Kondo screening by adjusting the microenvironment of the TM adatom. ${ }^{22-25}$ However, the thermochemical change associated with the Kondo spin-screening phenomenon and its implication to the chemical reactions catalyzed by the TM adatom are largely unexplored.

The main goal of this work is to investigate the possibility of utilizing the local spin moment on the SAC and the Kondo correlation effect to enhance further the reactivity. To this end, we propose a novel approach to heterogeneous single-atom catalysis by harnessing the evolution of Kondo spin-screening interaction during the reaction process.

\section{RESULTS AND DISCUSSION}

A new approach to heterogeneous catalysis: Reaction on a rink. Figure 1 depicts the free energy profile of a prototypical heterogeneous reaction with an SAC loaded on a metal support. If the metal support has the same temperature as the reactant species, which is high enough to activate the thermal catalytic reaction, the spin orientations of the metal's free electrons are randomized by the strong thermal fluctuations. As a result, the averaged spin-screening effect is expected to be negligibly small. In contrast, if the temperature of metal support is low enough so that the thermal fluctuations of free electrons are largely suppressed, and meanwhile the magnetic orbital of the SAC couples strongly to the metallic states, the Kondo spin-screening will occur spontaneously.

From the above analysis, lowering the temperature of metal support $\left(T_{\mathrm{s}}\right)$ will result in a reduction of the activation barrier associated with the heterogeneous catalytic reaction, if the spin-screening effect presents only in the transition state (TS) but not in the initial state (IS). An ideal scenario is that the local spin moment on the SAC is appreciable in the TS but absent in the IS; see Figure 1. Such a scenario may be realized by a rational design of the SAC and its microenvironment. Experimentally, it has been shown that the Kondo spin-screening can be switched on and off by chemical doping. ${ }^{23-25}$

A reduced barrier height usually means enhanced kinetics. Thus, with $T_{\mathrm{s}}$ being much lower than the temperature of reactant species $\left(T_{\mathrm{r}}\right)$, the accelerated heterogeneous reaction is reminiscent of skating on an ice rink. Therefore, we term the new approach to heterogeneous catalysis as "reaction on a rink" (ROAR). Compared to the conventional situation of $T_{\mathrm{s}}=T_{\mathrm{r}}$, the activation free

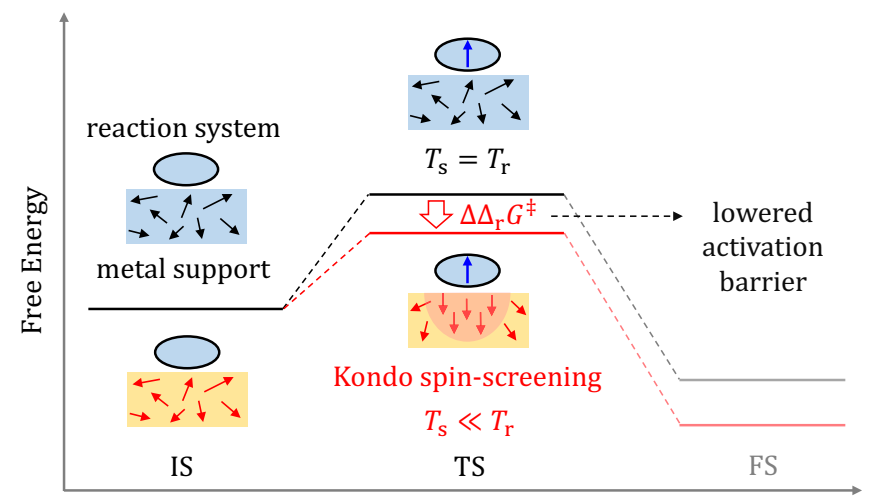

Reaction Coordinate

FIG. 1. Schematic illustration of the proposed ROAR approach to heterogeneous single-atom catalysis. The horizontal line segments indicate the relative free energies of the composite reaction system (reactant species and metal support) in the initial state (IS), transition state (TS), and final state (FS). The solid arrows represent the electronic spins on the $\mathrm{SAC}$ and in the metal support. With the temperature of metal support $\left(T_{\mathrm{s}}\right)$ being much lower than that of the reactant species $\left(T_{\mathrm{r}}\right)$, Kondo states form at the $\mathrm{SAC} /$ metal interface in the TS due to the spin-screening effect; see the main text for details. This leads to a reduction in the activation barrier $\left(\Delta \Delta_{\mathrm{r}} G^{\mp}\right)$, and hence accelerates the catalytic reaction.

energy in a ROAR with $T_{\mathrm{s}} \ll T_{\mathrm{r}}$ is reduced by a certain amount, as indicated by $\Delta \Delta_{\mathrm{r}} G^{\neq}$in Figure 1 . This is because the screening of the local spin is an exothermic process, which originates from the nonlocal electron correlation effect.

Since the spin-screening does not affect directly the atomic structure or charge state of the reactant species, the rate coefficient will remain largely unchanged upon the variation of $T_{\mathrm{s}}$. Therefore, by the Arrhenius formula the ROAR will give rise to a speedup factor $\gamma$ for the reaction rate $r$ as follows,

$$
\gamma \equiv \frac{r_{\mathrm{ROAR}}}{r} \simeq \exp \left(-\frac{\Delta \Delta_{\mathrm{r}} G^{\mp}}{R T_{\mathrm{r}}}\right),
$$

where $R$ is the gas constant. Obviously, the acceleration of reaction is more prominent with a more negative $\Delta \Delta_{\mathrm{r}} G^{\neq}$or a lower $T_{\mathrm{r}}$. Meanwhile, with the spins of free electrons aligning in the opposite direction to the local spin on the SAC, the spin configuration of the composite reaction system becomes more ordered, indicating a decrease in the total entropy.

To verify the practical feasibility of ROAR, in the following we propose theoretically a heterogeneous reaction which may be accelerated by the Kondo spin-screening effect. The reaction mechanism is scrutinized by carrying out first-principles-based calculations with a combined density functional theory ${ }^{26,27}$ and hierarchical equations of motion approach, ${ }^{28,29}$ see Methods for details.

A potential candidate of ROAR - CO oxidation catalyzed by Co@CoPc/Au(111). CO oxidation has 
a

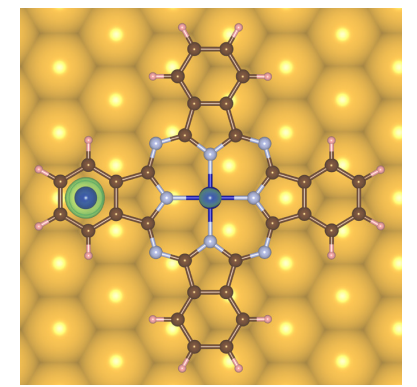

b

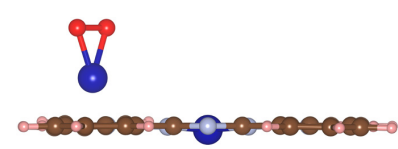

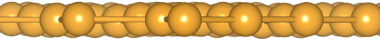

$\mathrm{Au}$

$\mathrm{Co} \odot \mathrm{N} \bullet \mathrm{C} \odot \mathrm{H}$
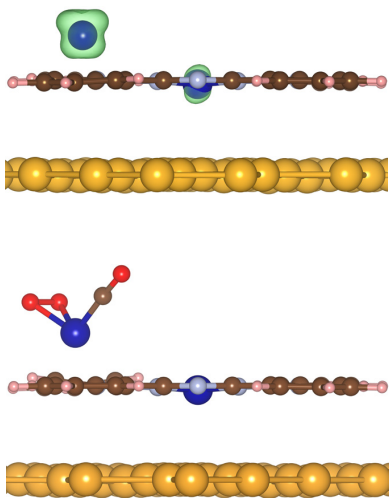

FIG. 2. Geometric structure of a potential SAC for CO oxidation via ROAR. (a) Top and side views of the SAC - Co@CoPc adsorbed on an $\mathrm{Au}(111)$ surface. The green isosurface represents the spin density of $0.08 \AA^{-3}$. (b) Structure of the SAC with a single $\mathrm{O}_{2}$ adsorbed onto the exposed Co atom, and that with the co-adsorption of an $\mathrm{O}_{2}$ and a CO.

been studied extensively in the field of heterogeneous catalysis. ${ }^{30-32}$ In particular, the use of single-atom and single-cluster catalysts has allowed $\mathrm{CO}$ oxidation to occur under low temperatures. ${ }^{33-35}$ For instance, the reaction temperature of $T_{\mathrm{r}}=200 \mathrm{~K}$ or even lower has been realized with dual-metal (Fe-Co) single sites dispersed on an N-doped carbon support, ${ }^{35} \mathrm{FeO}_{x}$ atomic layers deposited on a $\mathrm{Pt} / \mathrm{SiO}_{2}$ sample, ${ }^{33}$ and $\mathrm{Au}_{8}$ clusters bound to oxygen-vacancy F-center defects on an $\mathrm{Mg}(001)$ surface. ${ }^{34}$

Recently, we have successfully used cobalt phthalocyanine $(\mathrm{CoPc})$ molecules as molds to regularize the local spin states of Co atoms dispersed on an $\mathrm{Au}(111)$ surface. $^{22}$ It was found that a Co atom captured by a $\mathrm{CoPc}$ molecular mold locates either above or beneath an isoindole unit of the $\mathrm{Pc}$ ring. In the former case, the Co atom is exposed to the open air, and may thus act as an SAC for CO oxidation. Moreover, $\mathrm{CoPc}$ molecules could self-assemble into a superlattice layer on the gold support. ${ }^{36}$ Such a molecular layer may serve as a heat insulator, which helps to keep $T_{\mathrm{s}}$ much lower than $T_{\mathrm{r}}$ and hence facilitates the realization of ROAR. In the following, we demonstrate that the catalytic activity of $\mathrm{Co} @ \mathrm{CoPc} / \mathrm{Au}(111)$ toward $\mathrm{CO}$ oxidation could be further enhanced by utilizing the ROAR approach.

Adsorption of $\mathrm{O}_{2}$ and $\mathrm{CO}$ on $\mathrm{Co} @ \mathrm{CoPc} / \mathrm{Au}(111)$. Figure 2(a) depicts the optimized geometric structure and spin density distribution of the $\mathrm{Co} @ \mathrm{CoPc} / \mathrm{Au}(111)$ composite system. The spin-unpaired electrons reside predominantly on the $d_{\pi}$ orbitals of the Co atom, and the dumbbell-shaped spin density distribution is a result of strong $d_{\pi}-\pi$ bonding between the Co atom and the

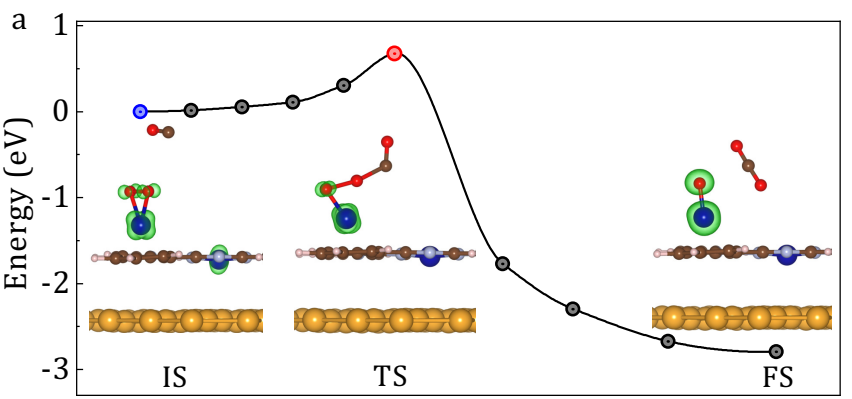

Reaction Coordinate

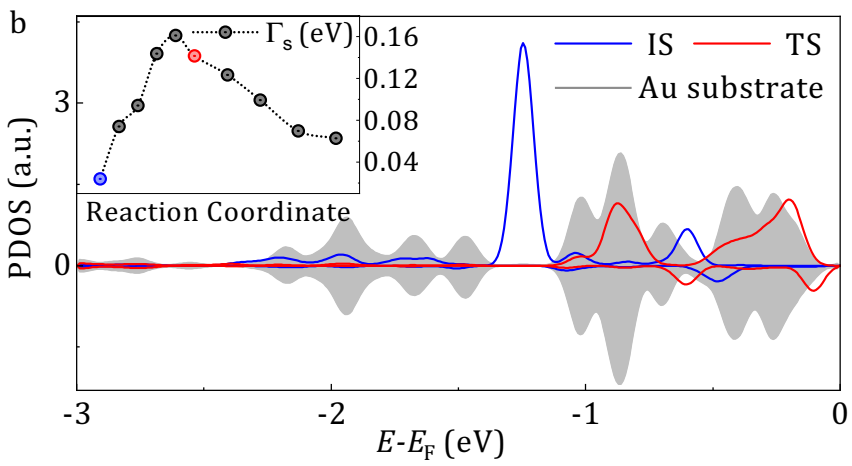

FIG. 3. Evolution of Kondo spin-screening along the ER pathway of $\mathrm{CO}$ oxidation. (a) Potential energy profile of the first $\mathrm{CO}$ oxidation step. The green isosurfaces represent the spin density of $0.14 \AA^{-3}$ in the IS, TS and FS. (b) PDOS of the magnetic $d$ orbital on the Co atom ( $d_{x z}$ in the IS and $d_{y z}$ in the TS) and the $s$ orbitals of gold substrate, where positive (negative) values correspond to the majority-spin (minority-spin) electrons. The inset shows the variation of $s$ - $d$ hybridization strength $\Gamma_{\mathrm{s}}$ along the reaction pathway. In particular, $\Gamma_{\mathrm{s}}=0.02 \mathrm{eV}$ and $0.14 \mathrm{eV}$ in the IS and TS, respectively.

nearby isoindole unit.

We then examine the adsorption of gaseous reactants onto an exposed Co atom and the associated thermochemical changes. The local geometries of adsorbate and adsorbent are displayed in Figure 2(b). Calculation predicts an adsorption energy of $-2.63 \mathrm{eV}$ for a single $\mathrm{O}_{2}$, which is lower by $0.25 \mathrm{eV}$ than that for a single CO. This suggests that the Co@CoPc-catalyzed CO oxidation could proceed by following the Eley-Rideal (ER) mechanism. Besides, the energetically more favorable $\mathrm{O}_{2}$ adsorption effectively prevents the $\mathrm{CO}$ poisoning of the SAC. ${ }^{37}$

The co-adsorption of a $\mathrm{CO}$ onto the exposed Co atom with a pre-adsorbed $\mathrm{O}_{2}$ further stabilizes the whole composite system by $0.53 \mathrm{eV}$, and the subsequent $\mathrm{CO}$ oxidation occurs via the Langmuir-Hinshelwood (LH) pathway. While the co-adsorption of $\mathrm{CO}$ is barrierless in the most ideal scenario, in an actual co-adsorption process the attachment of a $\mathrm{CO}$ is likely to experience a finite energy barrier (see Figure S1), depending on the position and orientation of the $\mathrm{CO}$ as it approaches the Co atom.

Being a paramagnetic molecule, the adsorbed $\mathrm{O}_{2}$ partially quenches the local spin moment on the exposed Co atom. Moreover, the formation of the $\mathrm{O}-\mathrm{O}$ bond has a 
profound influence on the local electronic structure of the Co atom, which in turn varies significantly the strength of Kondo spin-screening.

Reaction pathways for CO oxidation and evolution of Kondo spin-screening. The $\mathrm{CO}$ oxidation catalyzed by Co@CoPc/Au(111) could take both the ER and LH pathways. In either pathway, each adsorbed $\mathrm{O}_{2}$ oxidizes two $\mathrm{CO}$ molecules. The first oxidation is the rate-determining step (RDS), through which a $\mathrm{C}-\mathrm{O}$ bond is formed between one of the $\mathrm{O}$ atoms in the adsorbed $\mathrm{O}_{2}$ and a CO; see Figures 3(a), S2, and S3 for details. The RDS in both pathways have almost the same activation barrier heights. In the following, we will focus on the ER pathway, which is supposed to be more favorable because of the possible steric hindrance during the co-adsorption of $\mathrm{CO}$.

Figure 3(a) depicts the energy profile of RDS in the ER pathway. Unlike the ideal scenario of Figure 1, the Co atom in the IS has about the same local spin moment as that in the TS; see also TableS1. Nevertheless, as will be elucidated below, the TS indeed has a much stronger spin-screening effect than the IS, which is critical to the realization of ROAR.

Kondo correlation depends crucially on the strength of hybridization $\left(\Gamma_{\mathrm{s}}\right)$ between the magnetic $d$ orbital and the substrate metallic states. Regarding the projected density of states (PDOS) shown in Figure 3(b), the main peaks of the magnetic $d$ orbital on the Co atom barely overlap those of the gold $s$ orbitals in the IS. In contrast, the overlap is rather conspicuous in the TS. This indicates a much stronger $s$ - $d$ hybridization in the TS than in the IS (see also Figure S4), which is further affirmed by the evolution of $\Gamma_{\mathrm{s}}$ displayed in the inset of Figure 3(b).

Lowering of free energy barrier and acceleration of reaction rate with ROAR. We now explore the thermochemical change due to the prominent spinscreening effect in the TS and its consequence on the reaction kinetics. The activation barrier of RDS is $0.67 \mathrm{eV}$ under a normal reaction condition, which allows for a low reaction temperature of $T_{\mathrm{r}}=232 \mathrm{~K}$. Since the Co atom has a local spin of $S=1 / 2$ in the TS, a single-orbital Anderson impurity model (AIM) ${ }^{38}$ is employed to represent the $\mathrm{SAC} /$ metal composite, with which the magnetic $d$-orbital of Co and the metallic $s$-bands in the gold support are designated as the impurity and the reservoir, respectively.

The variation in activation barrier of RDS by implementing the ROAR approach can be evaluated by tracking the free energy change associated with the establishment of the $s$ - $d$ hybridization. Under the normal condition in which the metal support has a same temperature as that of the reactant species $\left(T_{\mathrm{r}}\right)$, the reaction energy

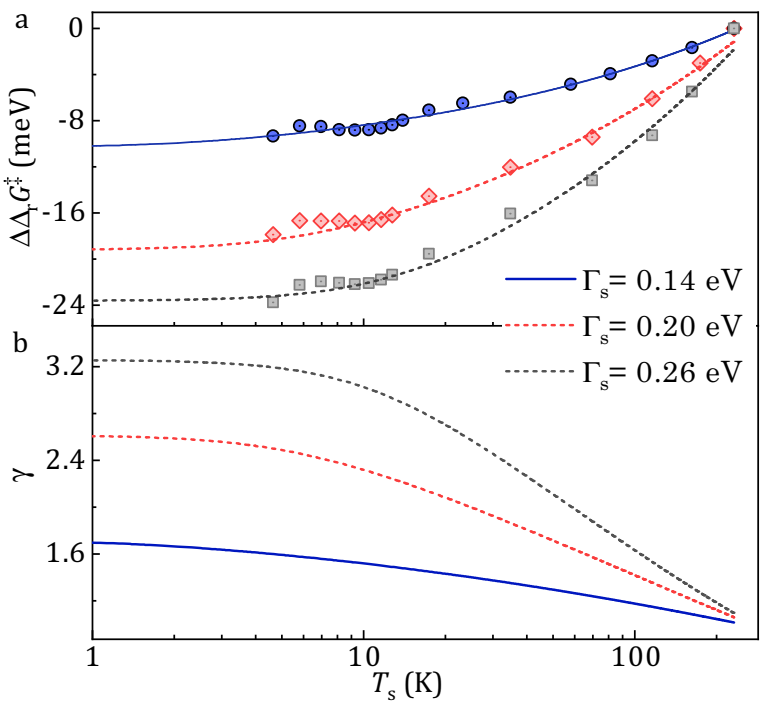

FIG. 4. Lowering of activation barrier and acceleration of reaction due to Kondo spin-screening effect in the TS. (a) $\Delta \Delta_{\mathrm{r}} G^{\neq}$and (b) speedup factor $\gamma$ versus $T_{\mathrm{s}}$, with $T_{\mathrm{r}}$ set to $232 \mathrm{~K}$. The scattered symbols in (a) represent the calculated data and the lines are fits to Eq. (5). The lines in (b) are the direct results of Eq. (1).

barrier is expressed as

$$
\begin{aligned}
\Delta_{\mathrm{r}} G^{\neq}= & G^{\mathrm{TS}}\left(T_{\mathrm{r}}\right)-G^{\mathrm{IS}}\left(T_{\mathrm{r}}\right) \\
= & {\left[G_{\mathrm{imp}}^{\mathrm{TS}}\left(T_{\mathrm{r}}\right)+G_{\mathrm{hyb}}^{\mathrm{TS}}\left(T_{\mathrm{r}}\right)+G_{\mathrm{s}}^{\mathrm{TS}}\left(T_{\mathrm{r}}\right)\right] } \\
& -\left[G_{\mathrm{imp}}^{\mathrm{IS}}\left(T_{\mathrm{r}}\right)+G_{\mathrm{hyb}}^{\mathrm{IS}}\left(T_{\mathrm{r}}\right)+G_{\mathrm{s}}^{\mathrm{IS}}\left(T_{\mathrm{r}}\right)\right] \\
= & {\left[G_{\mathrm{imp}}^{\mathrm{TS}}\left(T_{\mathrm{r}}\right)-G_{\mathrm{imp}}^{\mathrm{IS}}\left(T_{\mathrm{r}}\right)\right]+\left[G_{\mathrm{hyb}}^{\mathrm{TS}}\left(T_{\mathrm{r}}\right)-G_{\mathrm{hyb}}^{\mathrm{IS}}\left(T_{\mathrm{r}}\right)\right] . }
\end{aligned}
$$

Here, $G_{\mathrm{imp}}$ and $G_{\mathrm{s}}$ represent the Gibbs free energies of the isolated magnetic impurity and isolated reservoir, respectively; and $G_{\text {hyb }}$ denotes the free energy change before and after the $s$ - $d$ hybridization. While $G_{\text {imp }}$ varies as the reaction proceeds, $G_{\mathrm{S}}$ can be regarded as a constant.

By implementing the ROAR, $T_{\mathrm{s}}$ is varied to be much lower than $T_{\mathrm{r}}$, and the free energy barrier becomes

$$
\begin{aligned}
\Delta_{\mathrm{ROAR}} G^{\neq}= & {\left[G_{\mathrm{imp}}^{\mathrm{TS}}\left(T_{\mathrm{r}}\right)+G_{\mathrm{hyb}}^{\mathrm{TS}}\left(T_{\mathrm{s}}\right)+G_{\mathrm{s}}^{\mathrm{TS}}\left(T_{\mathrm{s}}\right)\right] } \\
& -\left[G_{\mathrm{imp}}^{\mathrm{IS}}\left(T_{\mathrm{r}}\right)+G_{\mathrm{hyb}}^{\mathrm{IS}}\left(T_{\mathrm{s}}\right)+G_{\mathrm{s}}^{\mathrm{IS}}\left(T_{\mathrm{s}}\right)\right] \\
= & {\left[G_{\mathrm{imp}}^{\mathrm{TS}}\left(T_{\mathrm{r}}\right)-G_{\mathrm{imp}}^{\mathrm{IS}}\left(T_{\mathrm{r}}\right)\right] } \\
& +\left[G_{\mathrm{hyb}}^{\mathrm{TS}}\left(T_{\mathrm{s}}\right)-G_{\mathrm{hyb}}^{\mathrm{IS}}\left(T_{\mathrm{s}}\right)\right] .
\end{aligned}
$$

Therefore, the reduction of free energy barrier by practising the ROAR is

$$
\Delta \Delta_{\mathrm{r}} G^{ \pm} \equiv \Delta_{\mathrm{ROAR}} G^{ \pm}-\Delta_{\mathrm{r}} G^{ \pm} \simeq G_{\mathrm{hyb}}^{\mathrm{TS}}\left(T_{\mathrm{s}}\right)-G_{\mathrm{hyb}}^{\mathrm{TS}}\left(T_{\mathrm{r}}\right) .
$$

Upon the last approximate equality of Eq. (4), we have used the fact that the $s$ - $d$ hybridization is rather weak in the IS, and thus $G_{\text {hyb }}^{\mathrm{IS}}\left(T_{\mathrm{s}}\right) \simeq G_{\text {hyb }}^{\mathrm{IS}}\left(T_{\mathrm{r}}\right)$.

It is interesting to find that the variation of $G_{\text {hyb }}^{\mathrm{TS}}$ with 
$T_{\mathrm{s}}$ fits accurately to an analytic formula of ${ }^{39}$

$$
G_{\mathrm{hyb}}^{\mathrm{TS}}\left(T_{\mathrm{s}}\right)=g_{a}\left[1+\left(\frac{T_{\mathrm{s}}}{T_{\mathrm{K}}}\right)^{2}\left(2^{\frac{1}{s}}-1\right)\right]^{s}+g_{b} .
$$

Here, $g_{a}$ and $g_{b}$ are two fitting parameters (see Table S6), $s=0.15$ is a constant, and $T_{\mathrm{K}}$ is an energy scale which characterizes the strength of Kondo correlation in the TS; see Note S3. ${ }^{40}$

As shown in Figure 4(a), $\Delta \Delta_{\mathrm{r}} G^{\ddagger}$ becomes more negative with the lowering of $T_{\mathrm{s}}$, which results in an increasing speedup factor $\gamma$. Specifically, $\Delta \Delta_{\mathrm{r}} G^{\neq}$reaches $-0.011 \mathrm{eV}$ with $T_{\mathrm{s}}$ reduced from $232 \mathrm{~K}$ to $1 \mathrm{~K}$, and the corresponding reaction rate is promoted by about 1.7 times. Although such an acceleration may seem minute, it is possible to enhance the efficacy of ROAR through rational design of the SAC and the metal support. For instance, if $\Gamma_{\mathrm{s}}$ in the TS could be enlarged to $0.20 \mathrm{eV}(0.26 \mathrm{eV})$, a more appreciable speedup of about 2.6 (3.3) times would be achieved; see Figure 4(b). In the literature, a $\Gamma_{\mathrm{s}}$ of $0.3 \mathrm{eV}$ has been reported for the $\mathrm{Co} / \mathrm{Cu}(100)$ composite. ${ }^{41}$

\section{CONCLUSIONS}

To summarize, we have proposed a novel approach to heterogeneous catalysis - the ROAR, which aims at enhancing catalytic activity by exploiting the quantum correlation between the local spin on the SAC and the electronic spins in the metal support. As a proof of concept, $\mathrm{CO}$ oxidation catalyzed by the $\mathrm{Co} @ \mathrm{CoPc} / \mathrm{Au}(111) \mathrm{com}-$ posite system is investigated. It is predicted that the ROAR will lead an appreciable acceleration of reaction rate. This work thus opens a new horizon for the field of heterogeneous single-atom catalysis by accentuating the potential usefulness of the spin degrees of freedom. In practice, since the ROAR favors an ultralow temperature of environment, it may be particularly valuable in outer space scenarios.

\section{METHODS}

A combined density functional theory (DFT) $)^{26,27}$ and hierarchical equations of motion (HEOM ${ }^{28,29}$ approach is employed to carry out the first-principles-based calculations. The DFT+HEOM approach has been successfully applied to describe the Kondo spin-screening effect in magnetic atom adsorbed on the metal support..$^{22,42,43}$

Density functional theory calculation. The Perdew-Burke-Ernzerholf (PBE) generalized gradient approximation implemented in the Vienna ab initio simulation package ${ }^{44}$ is employed, with the DFT-D3 method of Grimme ${ }^{45}$ used to improve the description of van der Waals interactions. The projected augmented wave method is adopted with the energy cutoff of $400 \mathrm{eV}$. A vacuum space of $16 \AA$ is adopted in the $z$-direction to avoid the artificial interaction between mirror images of the composite system. For geometry optimizations, a slab model including three layers of $\mathrm{Au}$ atoms is used to represent the $\mathrm{Au}(111)$ support, with each layer containing $56 \mathrm{Au}$ atoms. All the atoms except those in the bottom two $\mathrm{Au}$ layers are fully relaxed until the residual force on every atom is less than $0.02 \mathrm{eV} / \AA$. The convergence criterion for energy is set to $1 \times 10^{-5} \mathrm{eV}$. Because of the large size of the supercell, the $\Gamma$-point approximation is adopted.

The adsorption energy $E_{\text {ads }}$ of molecules is defined as

$$
E_{\mathrm{ads}}=E_{\mathrm{mol} / \mathrm{s}}-E_{\mathrm{mol}}-E_{\mathrm{s}},
$$

where $E_{\mathrm{mol} / \mathrm{s}}, E_{\mathrm{mol}}$ and $E_{\mathrm{s}}$ are the energies of the composite adsorption system, an isolated gas molecule, and a clean $\mathrm{Au}(111)$ support, respectively. The reaction pathway connecting the initial state (IS), transition state (TS) and final state (FS) is searched by using the climbing image nudged elastic band method, ${ }^{46}$ and the TS is settled with the force reduced below $0.05 \mathrm{eV} / \AA$.

Anderson impurity model. A single-orbital $\mathrm{AIM}^{38}$ is adopted to represent the magnetic $d$ orbital on the Co atom (the impurity) and the $s$ orbitals in the gold support (the reservoir). The total Hamiltonian is comprised of three parts:

$$
H_{\mathrm{AIM}}=H_{\mathrm{imp}}+H_{\mathrm{s}}+H_{\mathrm{hyb}},
$$

where $H_{\mathrm{imp}}$ and $H_{\mathrm{s}}$ are the Hamiltonian of the magnetic impurity and the gold support, respectively; and $H_{\text {hyb }}$ describes the $s$ - $d$ hybridization.

For CO oxidation catalyzed by the $\mathrm{Co} @ \mathrm{CoPc} / \mathrm{Au}(111)$ composite, both the IS and TS involve a primary magnetic orbital. The magnetic impurity is described by

$$
H_{\mathrm{imp}}=\epsilon_{d}\left(\hat{n}_{\uparrow}+\hat{n}_{\downarrow}\right)+U \hat{n}_{\uparrow} \hat{n}_{\downarrow} .
$$

Here, $\hat{n}_{\sigma}=\hat{d}_{\sigma}^{\dagger} \hat{d}_{\sigma}$, where $\hat{d}_{\sigma}^{\dagger}\left(\hat{d}_{\sigma}\right)$ creates (annihilates) a spin- $\sigma$ electron $(\sigma=\uparrow$ or $\downarrow)$ on the magnetic $d$ orbital of energy $\epsilon_{d}$; and $U$ is the on-site electron-electron Coulomb repulsion energy. The substrate is modeled by a reservoir of noninteracting electrons, i.e., $H_{\mathrm{s}}=\sum_{\sigma} \epsilon_{k} \hat{c}_{k \sigma}^{\dagger} \hat{c}_{k \sigma}$, where $\hat{c}_{k \sigma}^{\dagger}\left(\hat{c}_{k \sigma}\right)$ is the creation (annihilation) operator for a spin- $\sigma$ electron on the $k$ th metallic state. The impurity-reservoir hybridization has the form of $H_{\text {hyb }}=$ $\sum_{\sigma k} t_{k} \hat{c}_{k \sigma}^{\dagger} \hat{d}_{\sigma}+$ H.c., with $t_{k}$ being the coupling strength between the magnetic orbital and the $k$ th reservoir state.

The influence of electron reservoir on the magnetic impurity is fully characterized by the hybridization function, which assumes a Lorentzian form of $\Delta_{\mathrm{S}}(\omega) \equiv$ $\pi \sum_{k}\left|t_{k}\right|^{2} \delta\left(\omega-\epsilon_{k}\right)=\frac{\Gamma_{\mathrm{s}} W_{\mathrm{s}}^{2}}{\left(\omega-\Omega_{\mathrm{s}}\right)^{2}+W_{\mathrm{s}}^{2}}$. Here, $\Gamma_{\mathrm{s}}$ is the $s-$ $d$ hybridization strength, and $\Omega_{\mathrm{s}}\left(W_{\mathrm{s}}\right)$ is the band center (width) of the electron reservoir. The energetic parameters of the AIM are extracted from the results of DFT calculations $^{21,43,47}$ (in units of eV): $\epsilon_{d}=-0.6, U=1.48$, $\Gamma_{\mathrm{s}}=0.14, \Omega_{\mathrm{s}}=0$, and $W_{\mathrm{s}}=5.5$. In particular, $\Gamma_{\mathrm{s}}$ is extracted from the Green's function of the magnetic $d$ orbital of the Co atom. Details are given in the Note S1. 
Hierarchical equations of motion method. The HEOM method for fermionic environments ${ }^{28,48}$ implemented in the HEOM-QUICK program ${ }^{29}$ is employed to quantitatively characterize the Kondo spin-screening effect in the single-orbital AIM. To ensure a high accuracy for the AIM with a low $T_{\mathrm{s}}$, a recently developed Fano spectrum decomposition scheme $e^{49,50}$ is adopted to accurately unravel the reservoir correlation functions. The HEOM calculations are carried out with the truncation tier set to $L=4$.

The hybridization free energy in the TS is computed by using the thermodynamic integral formula: ${ }^{51}$

$$
G_{\text {hyb }}^{\mathrm{TS}}=\int_{0}^{1} \frac{\mathrm{d} \lambda}{\lambda}\left\langle H_{\mathrm{hyb}}\right\rangle_{\lambda}
$$

where $\langle\cdots\rangle$ denotes the expectation value taken at the stationary state of the impurity-reservoir composite with $H_{\text {hyb }}$ scaled by $\lambda$. Details are given in the Note S2 and Tables S2-S5.

\section{DATA AVAILABILITY}

The data that support the findings of this study are available within the paper and the Supplementary Information.

\section{CODE AVAILABILITY}

The HEOM-QUICK code utilized in this study is available at http://openquan.ustc.edu.cn/HEOM. *xz58@ustc.edu.cn

† jlyang@ustc.edu.cn

1 Wang, A.; Li, J.; Zhang, T. Heterogeneous single-atom catalysis. Nat. Rev. Chem. 2018, 2, 65-81.

${ }^{2}$ Lang, R.; Du, X.; Huang, Y.; Jiang, X.; Zhang, Q.; Guo, Y.; Liu, K.; Qiao, B.; Wang, A.; Zhang, T. Singleatom catalysts based on the metal-oxide interaction. Chem. Rev. 2020, 120, 11986-12043.

3 Wang, L.; Chen, W.; Zhang, D.; Du, Y.; Amal, R.; Qiao, S.; Wu, J.; Yin, Z. Surface strategies for catalytic $\mathrm{CO}_{2}$ reduction: from two-dimensional materials to nanoclusters to single atoms. Chem. Soc. Rev. 2019, 48, 53105349 .

${ }^{4}$ Li, X.; Yang, X.; Huang, Y.; Zhang, T.; Liu, B. Supported noble-metal single atoms for heterogeneous catalysis. Adv. Mater. 2019, 31, 1902031.

${ }^{5}$ Li, L.; Chang, X.; Lin, X.; Zhao, Z.-J.; Gong, J. Theoretical insights into single-atom catalysts. Chem. Soc. Rev. 2020, 49, 8156-8178.

${ }^{6}$ Wang, Y.; Su, H.; He, Y.; Li, L.; Zhu, S.; Shen, H.; Xie, P.; Fu, X.; Zhou, G.; Feng, C., et al. Advanced electrocatalysts with single-metal-atom active sites. Chem. Rev. 2020, 120, 12217-12314.

7 Kaiser, S. K.; Fako, E.; Manzocchi, G.; Krumeich, F.; Hauert, R.; Clark, A. H.; Safonova, O. V.; López, N.; Pérez-Ramírez, J. Nanostructuring unlocks high performance of platinum single-atom catalysts for stable vinyl chloride production. Nat. Catal. 2020, 3, 376-385.

8 Gong, Y.-N.; Zhong, W.; Li, Y.; Qiu, Y.; Zheng, L.; Jiang, J.; Jiang, H.-L. Regulating photocatalysis by spinstate manipulation of cobalt in covalent organic frameworks. J. Am. Chem. Soc. 2020, 142, 16723-16731.

9 Sun, S.; Shen, G.; Jiang, J.; Mi, W.; Liu, X.; Pan, L.; Zhang, X.; Zou, J.-J. Boosting oxygen evolution kinetics by $\mathrm{Mn}-\mathrm{N}-\mathrm{C}$ motifs with tunable spin state for highly efficient solar-driven water splitting. Adv. Energy Mater. 2019, 9, 1901505.

${ }^{10}$ Chen, P.; Tong, Y.; Wu, C.; Xie, Y. Surface/interfacial engineering of inorganic low-dimensional electrode materials for electrocatalysis. Acc. Chem. Res. 2018, 51, 2857-2866.
11 Shen, G.; Zhang, R.; Pan, L.; Hou, F.; Zhao, Y.; Shen, Z.; Mi, W.; Shi, C.; Wang, Q.; Zhang, X., et al. Regulating the spin state of $\mathrm{Fe}^{\mathrm{III}}$ by atomically anchoring on ultrathin titanium dioxide for efficient oxygen evolution electrocatalysis. Angew. Chem. Int. Ed. 2020, 59, 2313-2317.

12 Liu, J.-C.; Ma, X.-L.; Li, Y.; Wang, Y.-G.; Xiao, H.; Li, J. Heterogeneous $\mathrm{Fe}_{3}$ single-cluster catalyst for ammonia synthesis via an associative mechanism. Nat. Commun. 2018, 9, 1-9.

13 Liu, W.; Zhang, L.; Liu, X.; Liu, X.; Yang, X.; Miao, S.; Wang, W.; Wang, A.; Zhang, T. Discriminating catalytically active $\mathrm{FeN}_{x}$ species of atomically dispersed $\mathrm{Fe}-\mathrm{N}-\mathrm{C}$ catalyst for selective oxidation of the $\mathrm{C}-\mathrm{H}$ bond. J. Am. Chem. Soc. 2017, 139, 10790-10798.

14 Li, X.; Liu, L.; Ren, X.; Gao, J.; Huang, Y.; Liu, B. Microenvironment modulation of single-atom catalysts and their roles in electrochemical energy conversion. Sci. Adv. 2020, 6, eabb6833.

15 Ding, M.; Flaig, R. W.; Jiang, H.-L.; Yaghi, O. M. Carbon capture and conversion using metal-organic frameworks and MOF-based materials. Chem. Soc. Rev. 2019, 48, 2783-2828.

16 Ji, S.; Chen, Y.; Wang, X.; Zhang, Z.; Wang, D.; Li, Y. Chemical synthesis of single atomic site catalysts. Chem. Rev. 2020, 120, 11900-12314.

17 Qin, R.; Liu, K.; Wu, Q.; Zheng, N. Surface coordination chemistry of atomically dispersed metal catalysts. Chem. Rev. 2020, 120, 11810-11899.

18 Liu, K.; Zhao, X.; Ren, G.; Yang, T.; Ren, Y.; Lee, A. F.; Su, Y.; Pan, X.; Zhang, J.; Chen, Z., et al. Strong metalsupport interaction promoted scalable production of thermally stable single-atom catalysts. Nat. Commun. 2020, $11,1-9$.

19 Song, J.; Wei, C.; Huang, Z.-F.; Liu, C.; Zeng, L.; Wang, X.; Xu, Z. J. A review on fundamentals for designing oxygen evolution electrocatalysts. Chem. Soc. Rev. 2020, 49, 2196-2214.

${ }^{20}$ Kondo, J. Resistance minimum in dilute magnetic alloys. Prog. Theor. Phys. 1964, 32, 37.

21 Zhao, A. D.; Li, Q. X.; Chen, L.; Xiang, H. J.; Wang, W. H.; Pan, S.; Wang, B.; Xiao, X. D.; Yang, J. L.; 
Hou, J. G.; Zhu, Q. S. Controlling the Kondo effect of an adsorbed magnetic ion through its chemical bonding. Science 2005, 309, 1542.

${ }^{22}$ Li, X.; Zhu, L.; Li, B.; Li, J.; Gao, P.; Yang, L.; Zhao, A.; Luo, Y.; Hou, J.; Zheng, X., et al. Molecular molds for regularizing Kondo states at atom/metal interfaces. Nat. Commun. 2020, 11, 1-8.

${ }^{23}$ Liu, L. W.; Yang, K.; Jiang, Y. H.; Song, B. Q.; Xiao, W. D.; Li, L. F.; Zhou, H. T.; Wang, Y. L.; Du, S. X.; Ouyang, M.; Hofer, W. A.; Castro Neto, A. H.; Gao, H. J. Reversible single spin control of individual magnetic molecule by hydrogen atom adsorption. Sci. Rep. 2013, 3, 1210.

24 Xie, L.; Lin, H.; Zhang, C.; Li, J.; Merino-Díez, N.; Friedrich, N.; Bouju, X.; Li, Y.; Pascual, J. I.; Xu, W. Switching the spin on a Ni trimer within a metal-organic motif by controlling the on-top bromine atom. ACS Nano 2019, 13, 9936-9943.

${ }^{25}$ Krull, C.; Robles, R.; Mugarza, A.; Gambardella, P. Siteand orbital-dependent charge donation and spin manipulation in electron-doped metal phthalocyanines. Nat. Mater. 2013, 12, 337-343.

${ }^{26}$ Kohn, W.; Sham, L. J. Self-consistent equations including exchange and correlation effects. Phys. Rev. 1965, 140, A1133.

27 Hohenberg, P.; Kohn, W. Inhomogeneous electron gas. Phys. Rev. 1964, 136, B864.

28 Jin, J. S.; Zheng, X.; ; Yan, Y. J. Exact dynamics of dissipative electronic systems and quantum transport: Hierarchical equations of motion approach. J. Chem. Phys. 2008, 128, 234703.

29 Ye, L. Z.; Wang, X. L.; Hou, D.; Xu, R. X.; Zheng, X.; Yan, Y. J. HEOM-QUICK: a program for accurate, efficient, and universal characterization of strongly correlated quantum impurity systems. WIREs Comput. Mol. Sci. 2016, 6, 608 .

${ }^{30}$ Al Soubaihi, R. M.; Saoud, K. M.; Dutta, J. Critical review of low-temperature $\mathrm{CO}$ oxidation and hysteresis phenomenon on heterogeneous catalysts. Catalysts 2018, 8, 660.

31 Ackermann, M.; Pedersen, T.; Hendriksen, B.; Robach, O.; Bobaru, S.; Popa, I.; Quiros, C.; Kim, H.; Hammer, B.; Ferrer, S., et al. Structure and reactivity of surface oxides on $\mathrm{Pt}(110)$ during catalytic CO oxidation. Phys. Rev. Lett. 2005, 95, 255505.

32 Xie, X.; Li, Y.; Liu, Z.-Q.; Haruta, M.; Shen, W. Low-temperature oxidation of $\mathrm{CO}$ catalysed by $\mathrm{Co}_{3} \mathrm{O}_{4}$ nanorods. Nature 2009, 458, 746-749.

${ }^{33}$ Cao, L.; Liu, W.; Luo, Q.; Yin, R.; Wang, B.; Weissenrieder, J.; Soldemo, M.; Yan, H.; Lin, Y.; Sun, Z., et al. Atomically dispersed iron hydroxide anchored on $\mathrm{Pt}$ for preferential oxidation of $\mathrm{CO}$ in $\mathrm{H}_{2}$. Nature 2019, 565, 631635 .

34 Yoon, B.; Häkkinen, H.; Landman, U.; Wörz, A. S.; Antonietti, J.-M.; Abbet, S.; Judai, K.; Heiz, U. Charging effects on bonding and catalyzed oxidation of $\mathrm{CO}$ on $\mathrm{Au}_{8}$ clusters on MgO. Science 2005, 307, 403-407.

35 Wang, J.; You, R.; Zhao, C.; Zhang, W.; Liu, W.; Fu, X.P.; Li, Y.; Zhou, F.; Zheng, X.; Xu, Q., et al. N-coordinated dual-metal single-site catalyst for low-temperature CO oxidation. ACS Catal. 2020, 10, 2754-2761.

${ }^{36}$ Zhao, Y.; Li, B.; Yin, R.; Wang, Y.; Yang, J.; Zhang, Z.; Wang, B. Spin selection rule in single-site catalysis of molecular oxygen adsorption on transition-metal phthalo- cyanines. J. Phys. Chem. C 2019, 123, 28158-28167.

37 Li, Y.; Sun, Q. The superior catalytic CO oxidation capacity of a Cr-phthalocyanine porous sheet. Sci. Rep. 2014, 4, 4098.

38 Anderson, P. W. Localized magnetic states in metals. Phys. Rev. 1961, 124, 41.

39 Goldhaber-Gordon, D.; Göres, J.; Kastner, M.; Shtrikman, H.; Mahalu, D.; Meirav, U. From the Kondo regime to the mixed-valence regime in a single-electron transistor. Phys. Rev. Lett. 1998, 81, 5225.

40 Žitko, R.; Bonča, J. Multiple-impurity Anderson model for quantum dots coupled in parallel. Phys. Rev. B 2006, 74, 045312.

41 Choi, D.-J.; Rastei, M.; Simon, P.; Limot, L. Conductancedriven change of the Kondo effect in a single cobalt atom. Phys. Rev. Lett. 2012, 108, 266803.

42 Wang, X. L.; Yang, L. Q.; Ye, L. Z.; Zheng, X.; Yan, Y. J. Precise control of local spin states in an adsorbed magnetic molecule with an STM tip: theoretical insights from firstprinciples-based simulation. J. Phys. Chem. Lett. 2018, 9, 2418.

43 Wang, Y.; Zheng, X.; Li, B.; Yang, J. L. Understanding the Kondo resonance in the d-CoPc/Au (111) adsorption system. J. Chem. Phys. 2014, 141, 084713.

44 Perdew, J. P.; Burke, K.; Ernzerhof, M. Generalized gradient approximation made simple. Phys. Rev. Lett. 1996, 77, 3865.

45 Grimme, S.; Antony, J.; Ehrlich, S.; Krieg, H. A consistent and accurate ab initio parametrization of density functional dispersion correction (DFT-D) for the 94 elements H-Pu. J. Chem. Phys. 2010, 132, 154104.

46 Henkelman, G.; Uberuaga, B. P.; Jónsson, H. A climbing image nudged elastic band method for finding saddle points and minimum energy paths. J. Chem. Phys. 2000, 113, 9901-9904.

47 Hou, D.; Wang, R. L.; Zheng, X.; Tong, N. H.; Wei, J. H.; Yan, Y. J. Hierarchical equations of motion for an impurity solver in dynamical mean-field theory. Phys. Rev. B 2014, 90, 045141.

${ }^{48}$ Li, Z. H.; Tong, N. H.; Zheng, X.; Hou, D.; Wei, J. H.; $\mathrm{Hu}$, J.; Yan, Y. J. Hierarchical Liouville-space approach for accurate and universal characterization of quantum impurity systems. Phys. Rev. Lett. 2012, 109, 266403.

49 Cui, L.; Zhang, H.-D.; Zheng, X.; Xu, R.-X.; Yan, Y. J. Highly efficient and accurate sum-over-poles expansion of Fermi and Bose functions at near zero temperatures: Fano spectrum decomposition scheme. J. Chem. Phys. 2019, $151,024110$.

${ }^{50}$ Zhang, H.-D.; Cui, L.; Gong, H.; Xu, R.-X.; Zheng, X.; Yan, Y. Hierarchical equations of motion method based on Fano spectrum decomposition for low temperature environments. J. Chem. Phys. 2020, 152, 064107.

51 Gong, H.; Wang, Y.; Zhang, H.-D.; Qiao, Q.; Xu, R.X.; Zheng, X.; Yan, Y. Equilibrium and transient thermodynamics: a unified dissipaton-space approach. J. Chem. Phys. 2020, 153, 154111.

\section{ACKNOWLEDGMENTS}

The support from the National Key Research and Development Program of China (Grant No. 2016YFA0200600), the National Natural Science Founda- 
tion of China (Grant Nos. 21973086 and 21688102), the Strategic Priority Research Program of Chinese Academy of Sciences (Grant No. XDB36000000), and the Anhui Initiative in Quantum Information Technologies (Grant No. AHY090000) is greatly appreciated. The computational resources are provided by the Supercomputing Center of University of Science and Technology of China and Tianjin Supercomputer Center.

\section{AUTHOR CONTRIBUTIONS}

X.Z. and J.Y. conceived the project. X.L. and X.Z. conducted the theoretical calculations. X.L., X.Z., J.Y., H.G. and Q.Z. analyzed the theoretical data. X.L., X.Z.,
J.Y. and B.W. co-wrote the paper. All authors discussed the results and commented on the manuscript.

\section{COMPETING INTERESTS}

The authors declare no competing interests.

\section{ADDITIONAL INFORMATION}

Supplementary information is available for this paper at https://doi.org. 